\title{
Digital Health Record and Prescription
}

\author{
A.P. Mohod ${ }^{1 *}$, A. Pankar ${ }^{2}$, A Mohite ${ }^{3}$, A. Dondalkar ${ }^{4}$, A. Kadhe ${ }^{5}$, Manish Naik ${ }^{6}$ \\ ${ }^{1 *}$ Dept. of CSE, Priyadarshini J.L. College of Engg., (Rashtrasant Tukadoji Maharaj Nagpur University), Nagpur, India \\ ${ }^{2}$ Dept. of CSE, Priyadarshini J.L. College of Engg.,(Rashtrasant Tukadoji Maharaj Nagpur University), Nagpur, India \\ ${ }^{3}$ Dept. of CSE, Priyadarshini J.L. College of Engg.,(Rashtrasant Tukadoji Maharaj Nagpur University), Nagpur, India \\ ${ }^{4}$ Dept. of CSE, Priyadarshini J.L. College of Engg.,(Rashtrasant Tukadoji Maharaj Nagpur University), Nagpur, India \\ ${ }^{5}$ Dept. of CSE, Priyadarshini J.L. College of Engg.,(Rashtrasant Tukadoji Maharaj Nagpur University), Nagpur, India \\ ${ }^{6}$ Dept. of CSE, Priyadarshini J.L. College of Engg.,(Rashtrasant Tukadoji Maharaj Nagpur University), Nagpur, India \\ *Corresponding Author: mohod.ashish@gmail.com, Tel.: +91-8668598879
}

Available online at: www.isroset.org

Received 22/Dec/2017, Revised 9/Jan/2018, Accepted 25/Jan/2018, Online 28/Feb/2018

\begin{abstract}
A majority of patients face problems due to negligence of officials. And to maintain all the records in registers or papers for Person, Doctor or even Government is a very complicated task. The hand written prescriptions can be sometimes difficult for shopkeeper or Chemist to understand and finally results in giving the wrong medicines that causes more harm than help. And so there is need to maintain the health record digitally to reduce the doctor's task and help the patients to understand their health record. In this project, we are developing a cloud based application which works as SaaS that will store every registered person's lifetime health record that can be retrievable anytime, anywhere. This application will be used by the doctors, where they will be able to access patient's medical history by their ID and diagnose the current sickness accordingly. Doctors can read, write and edit Patient's reports and prescriptions will be automatically generated which will reduce the chances of misunderstanding with the chemists.
\end{abstract}

Keywords - Cloud Storage, Patient Health Record, SaaS, Doctors, Patients

\section{INTRODUCTION}

This project is mainly focused on the medical data of the patients. Patient consults doctors and gets a report that is checked by doctors then a particular medication is given to the patient. The reporting process required from the doctors is very time consuming. If the patient is physically disabled then he/she has to struggle at these things. Because of these problems, we've thought of an idea that a unique id will be given to the patients so that doctors can access their reports, if needed. After examination, all the reports and prescription will be uploaded to the patient's id. The patient, then, can get the medicines whenever they want to. In case, the patient lost his hardcopy of the reports then it can be retrieved from our portal. Let's take a scenario," A patient is suffering from a disease so he consulted a doctor and got his report online. The patient needs to go out of the city for some business purpose and there he got ill. If he consults a doctor, he can show his previous medical reports to the doctors so that they can examine him properly by keeping allergic medications aside". For this type of scenario, this system comes in handy.

The organization of the paper is as follows, Section I contains the introduction of Digital Health Record and Prescription, Section II contains the related work of Digital
Health Record, Section III contains the architecture and proposed system of Digital Health Record, Section IV concludes research work with future directions.

\section{LITERATURE SURVEY}

E. Coiera, Digital Health Records are contemplated as a valuable source for supporting the provision of high quality healthcare. However, the purpose of DHR is mixed, the proof of their efficiency and previous studies have generally considered DHR as a binary factor and have not considered the availability and use of specific DHR features. Main objective was to evaluate the relationship between the use of a DHR and the use of specific DHR features with quality of care.

Although when considering past studies, there was no indicative relationship between use of DHR as a binary factor and quality measure based performances. In spite of the use of selected features of DHR by physicians with primary care was associated with higher performance on certain quality measures [1]. All these results suggest to take a step to increase health care quality and should focus on increasing the adoption of robust DHR systems and increasing the application of attributes rather than simply deploying a DHR notwithstanding of functionality. 
Centre for Biomedical Informatics, Temple University, Philadelphia, PA 19122, USA, Phenotype discoveries on Digital Health Records (DHR) data have recently started taking benefits across many aspects of clinical practice that are data driven. In their method, they'd made a very large DHR database that contained more than a million inpatient cases into a low dimensional space. This embedding allows for an effective distinguishing the diseases into more alike categories, an important task of discovering disease types for precision medicine. In particular, many diseases have heterogeneous nature. For example, a systemic and progressive inflammation, can be caused by so many factors, and can have multiple demonstrations on human organs. Understanding such heterogeneity of the disease can give hints to many important issues related to sepsis, including early diagnosis and treatment, which is of a huge significance as sepsis was one of the crucial reasons of demise in the hospitals in the United States [2]. This study analyses state of the art embedding models that have had huge success in many fields, applying them to disease embedding from DHR databases.

Sebastien Haneuse, DHR data can contribute a reasonable and a large sample size over a long time session as compared to devoted clinical studies; however, it is hard to recall that EHR data were not collected to assist particular tasks. Haneuse inspired researchers to compare the available digital health data to the data that would result from an allocated study, saying that observational data probably do not have proportionate standards. There are supplementary challenges to use EHR system, such as taking out text-based information contained in clinician notes, unreliable information, linking patient records across databases, and confounding bias [3]. In the context of the antidepressant study, EHR data preferably contained statistics on weight at baseline and 2 years for each patient, but now in reality less than 25 percent of patients had those statistics together, leaving 75 percent of patients with inadequate information to compute the primary outcome[4].

Isaac S. Kohane, if genomic studies are to be a impersonally admissible and suitable assessment of the relationship between genetics and health status, whether for common or common variants, efficient ways must be built to measure both the genetic variation and the phenotypic attribute of mass population. The acquisition of electronic health records, used by practitioner to record clinical care, is getting widespread and recent studies indicate that they can be effectively appointed for genetic research using the collection of data while maintaining privacy [5].

\section{ArChitecture Of Proposed System}

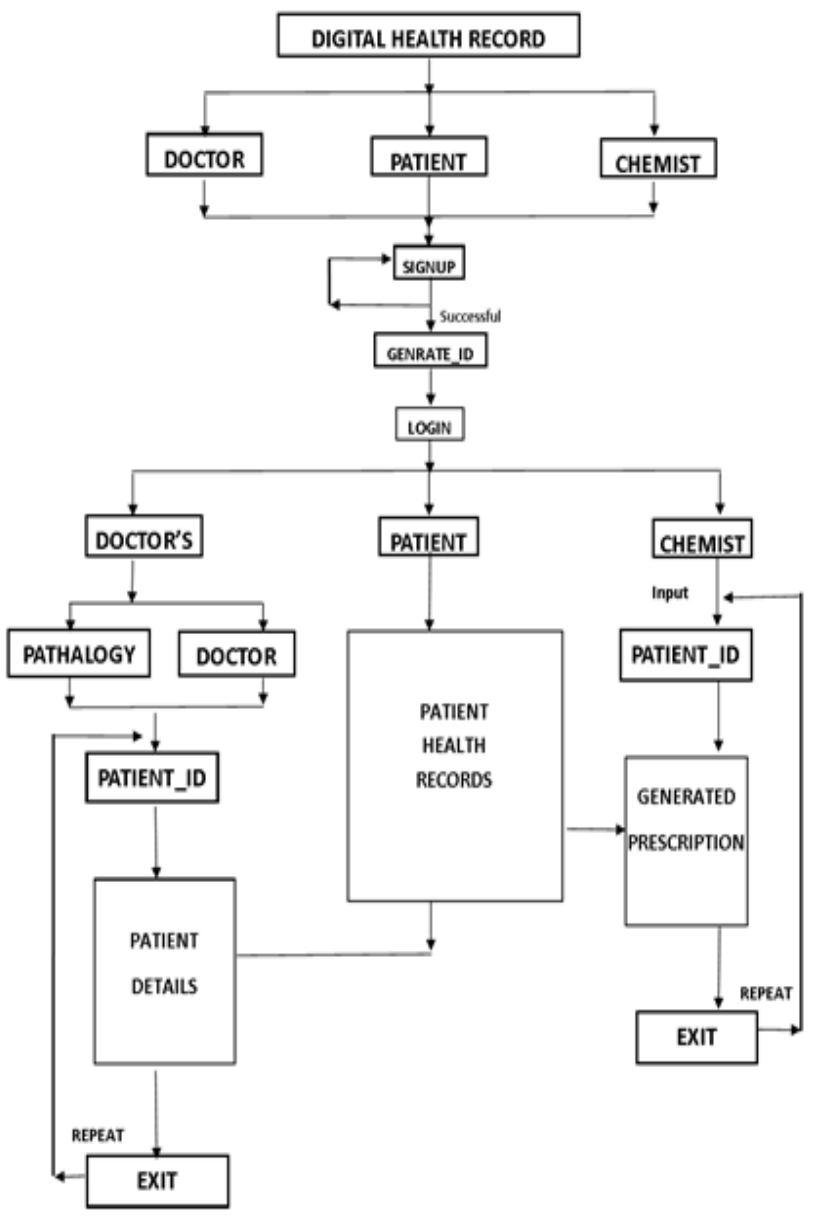

System

Fig 1: Architecture of Digital Health Record

To develop a Web Application we are using front end as Bootstrap Technology which is in very popular now a days for Responsive web applications. And for backend Salesforce cloud which provide more advance automated feature for Storing, Retrieve and Update patient health data. Salesforce Cloud improves the Data Quality and Management of the Customers. All CRMs have one core function that is to manage the data about customers. Salesforce gives an easy interface to the organizations so that the customer data can be easily managed by the providing companies with a host of easy-to-use options to input. There are various options in the Data Loader like Insert, Update, Upsert, Delete, and hard delete which allows user to add new records, modify or change or delete existing records. "Upsert" is an operation that is a combination of Inserting and Updating record. If a record in a specific file matches an existing record, the existing record is updated with the values in your file. And new record is created when no match is found. When hard 
delete operation is performed on records, the deleted records are not stored in the Recycle Bin.

The reason to use Bootstrap Technology is

i. Speed of Development

It is extremely an easy and speedy procedure to begin with Bootstrap. Bootstrap is very versatile too. Bootstrap can be utilized along with HTML, LESS, CSS or with SaaS.

ii. Responsiveness

Mobile devices, nowadays, continue to grow because of "On-the-Go" system as advancing in technology so the idea of responsive page came into existence. With the help of Bootstrap, websites can be easily adjusted to any screen resolution.

\section{CONCLUSION AND FUTURE SCOPE}

This research paper gives the solution to the problem of using paper records and prescriptions. This model also explains to keep track of a person's health record in a systematic way. Since, we are using the system in a cloud environment, therefore security issues and storage issues will not affect the system. This System will overcome the drawbacks of traditional approach in health record system.

The Digital Health Record System will ensure the patient's health data for long time and in future a system can be developed so that it will work on the whole country.

\section{REFERENCES}

[1]. E. Coiera, The Guide to Health Informatics (2nd Edition). Arnold, London, October 2003. [Chapter 10: The electronic medical record, p111-123] [See also: Chapter 9: Information management systems p101-110].

[2]. Center for Data Analytics and Biomedical Informatics, Temple University, Philadelphia, PA 19122, USA.

[3]. Sebastien Haneuse, Harvard University, A general framework for selection bias due to missing data in electronic health records-based research

[4]. https://www.nap.edu/read/24654/chapter/5\#37

[5]. Isaac S. Kohane Using electronic health records to drive discovery in disease genomics http://www.nature.com/nrg/journal/v12/n6/full/nrg2 999.html?foxtrotcallback=true

\section{Authors Profile}

Ashish Mohod received the Bachelor of degree from U.C.O.E, Umred, and State-Maharashtra, India. He recieved MTech. Degree in Computer Science and Engineering from Bhagwati Chaturvedi College of Engineering, Nagpur. Maharashtra, India. He was a Software developer between 2005 to 2009 . He has presented more than 5 papers, in national conferences and publishes many international journal papers. He is currently working as a lecturer in Priyadarshini J.L. college of Engineering, Nagpur. He has organized workshops, state/national project events, seminars in various organizations. His interest of research is in Effective

Materialized View Selection and Maintenance process.

Abhishek Pankar is pursuing his Bachelor of Engineering in Computer Science from Priyadarshini J.L. College of Engineering, Rashtrasant Tukadoji Maharaj Nagpur University, Nagpur-440009, India

Ajinkya Dondalkar is pursuing his Bachelor of Engineering in Computer Science from Priyadarshini J.L. College of Engineering, Rashtrasant Tukadoji Maharaj Nagpur University, Nagpur-440009, India

Aditya Mohite is pursuing his Bachelor of Engineering in Computer Science from Priyadarshini J.L. College of Engineering, Rashtrasant Tukadoji Maharaj Nagpur University, Nagpur-440009, India

Ajinkya Kadhe is pursuing his Bachelor of Engineering in Computer Science from Priyadarshini J.L. College of Engineering, Rashtrasant Tukadoji Maharaj Nagpur University, Nagpur-440009, India

Manish Naik is pursuing his Bachelor of Engineering in Computer Science from Priyadarshini J.L. College of Engineering, Rashtrasant Tukadoji Maharaj Nagpur University, Nagpur-440009, India

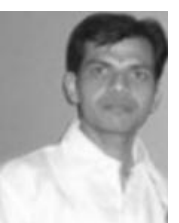
.
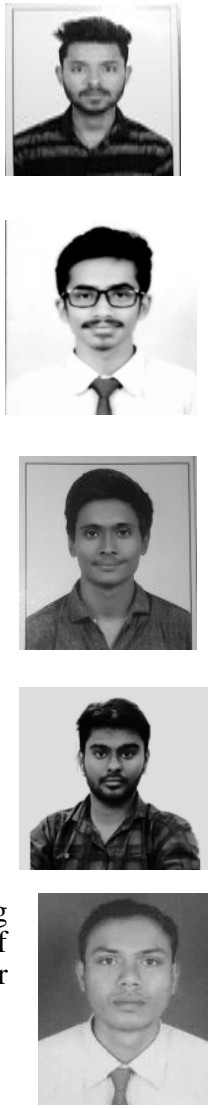